\title{
BMJ Open Sick leave and medication use in pregnancy: a European web-based study
}

\author{
Bich Thuy Truong, ${ }^{1}$ Angela Lupattelli, ${ }^{1}$ Petter Kristensen, ${ }^{2,3}$ Hedvig Nordeng ${ }^{1,4}$
}

\begin{abstract}
To cite: Truong BT, Lupattelli A, Kristensen $\mathrm{P}$, et al. Sick leave and medication use in pregnancy: a European web-based study. BMJ Open 2017;7:e014934. doi:10.1136/ bmjopen-2016-014934

- Prepublication history and additional material for this paper are available online. To view these files please visit the journal online (http://dx.doi. org/10.1136/bmjopen-2016014934).
\end{abstract}

Received 28 0ctober 2016 Revised 17 May 2017 Accepted 20 June 2017

\section{(a) CrossMark}

${ }^{1}$ PharmacoEpidemiology and Drug Safety Research Group, Department of Pharmacy, School of Pharmacy, University of Oslo, Oslo, Norway

${ }^{2}$ Department of Occupational Medicine and Epidemiology, National Institute of Occupational Health, 0slo, Norway

${ }^{3}$ Preventive Medicine and Epidemiology Research Group, Institute of Health and Society, University of Oslo, Oslo, Norway ${ }^{4}$ Department of Child Health, Norwegian Institute of Public Health, Oslo, Norway

Correspondence to Bich Thuy Truong; b.t.h.truong@farmasi.uio.no

\section{ABSTRACT}

Background and objective A comparison of sick leave in pregnancy between countries is difficult as most studies have been conducted in single countries in Scandinavia. The objective of this study was to explore patterns of and reasons for sick leave during pregnancy on a multinational level, focusing on medication use but also differences in sick leave policies.

Design and setting Cross-sectional, web-based study in 12 European countries from October 2011 to February 2012. Data were collected via an electronic questionnaire. Participants Pregnant women and mothers of children under the age of 1 year.

Primary outcome measure Sick leave prevalence in pregnancy.

Results Of 6686 women included, 3385 (50.6\%) had been on sick leave during pregnancy. The rates of sick leave varied across countries, ranging from $31.7 \%-34.8 \%$ in Sweden and the UK to $62.4 \%-71.3 \%$ in Norway, Serbia, Croatia and Poland. The most common reasons for being on sick leave were pregnancy complications $(26.5 \%)$; pain in the neck, back or pelvic girdle (16.2\%); and nausea and vomiting (NVP, 16.0\%). Women using medications for acute illnesses were more likely to be on sick leave than their non-medicated counterparts, while an opposite trend was observed for women with chronic disorders, where nonmedicated women were more likely to be on sick leave. Women from countries with 'low' sick leave policies were less likely to have extensions of sick leaves compared with women from countries with 'medium' policies (adjusted OR $0.63,95 \% \mathrm{Cl} 0.49$ to 0.82 ).

Conclusion The rates of sick leave in pregnancy vary greatly across European countries. Women using medications were more likely to be on sick leave, especially for acute illnesses. The differences in sick leave patterns across countries only partially reflected differences in sick leave policies, which implies that sick leave in pregnancy is also affected by other national differences.

\section{INTRODUCTION}

Multiple studies conducted in Scandinavia have identified generally high rates of sick leave among pregnant women (range $48.0 \%-67.7 \%),{ }^{1-5}$ and pregnancy-related conditions, such as back pain and nausea and vomiting (NVP), were the most common reasons for sick leave. ${ }^{3467}$ Sick leave in pregnancy may also be required when there are risky occupational exposures or work-related
Strengths and limitations of this study

- Uniform data collection methodology, allowing intercountry comparisons of sick leave in pregnancy.

- New insights into patterns of sick leave in pregnancy for countries outside Scandinavia, as well as into the impact of medication of acute and chronic disorders on sick leave.

- No detailed information about sick leave, that is, exact timing, duration and whether it was part time or full time.

- A web-based survey as a study method impedes the calculation of a conventional response rate and may cause selection bias of the target population.

- Self-reported data used for dependent and independent variables.

duties that may negatively impact pregnancy outcomes. ${ }^{8-12}$ Interestingly, research on sick leave in pregnancy is almost non-existent for countries outside Scandinavia, which makes intercountry comparisons challenging. When considering the high extent of sick leave seen in the Scandinavian countries, it is crucial to gain knowledge about patterns and factors associated with sick leave during pregnancy to initiate preventive measures. Such knowledge would not only be of huge economic interest for society, but it would also be beneficial for clinicians who encounter pregnant women in antenatal care. Elucidating patterns of sick leave among pregnant women in other European countries can also contribute to a better understanding of maternal health across countries. National differences in work participation by women of fertile age, as well as the thresholds and attitudes towards sick leave, may differ across countries despite the same conditions affecting pregnant women. ${ }^{13} 14$

The concept of paid sick leave is included in the welfare systems of most Western countries and it is intended to provide employees with financial protection during sickness and disability. ${ }^{15}$ However, the qualifications for receiving sick leave benefits vary greatly between countries. The WHO described the concept of paid sick leave from a global perspective, revealing that 145 countries 
provide paid sick leave and the wage replacement ranges from lump sums, in $14.0 \%$ of the countries, to $100.0 \%$ of wages in $21.0 \%$ of the countries. ${ }^{15}$

Many medical conditions occur during pregnancy and pregnant women may need medical treatment to ensure maternal-fetal health. ${ }^{16}$ However, pregnant women tend to overestimate the teratogenic risk associated with medications ${ }^{17}$ which can result in untreated conditions due to fear of harming the unborn child. ${ }^{18}$ As some untreated maternal conditions, for example, diabetes and epilepsy, can pose a risk to the fetus, it is essential that pregnant women are empowered to make safe decisions about treatment options in pregnancy. ${ }^{16}$ Sociodemographic and lifestyle factors, such as age, gender and education ${ }^{19}$; self-reported health ${ }^{20}$ and work-related conditions ${ }^{41-23}$ are potential determinants of sick leave in general, but no previous studies have investigated the extent of sick leave in pregnancy with respect to maternal medication use. Therefore, the role of medication use in sick leave during pregnancy remains elusive.

The objective of this study was to describe patterns of and reasons for sick leave in pregnancy on a multinational European level, focusing on maternal illnesses and related medication use. In addition, we explored sick leave in relation to the differences in European sick leave policies.

\section{METHODS}

\section{Study design, data collection and study population}

This is a substudy of the 'Multinational Medication Use in Pregnancy Study', a cross-sectional, web-based study carried out in Europe, North and South America and Australia from October 2011 to February 2012, with the purpose to investigate patterns of medication use in pregnancy. The study has been described in detail elsewhere. ${ }^{24}$ In brief, member countries of the European Network of Teratology Information Services, Organization of Teratology Information Specialist in North and South America, Mothersafe in Australia and European institutions conducting public health research were invited to take part in the project. Of these, 18 countries participated. Data were collected via an anonymous, self-administrated, questionnaire (www.questback.com), accessible in each participating country for 2 months in the period mentioned above. The full questionnaire has previously been published. ${ }^{24}$ An invitation to participate in the study (presented as banner/ads) was available on two to three national pregnancy-related web pages and/ or social networks, selected according to the number of daily users in each participating country. Pregnant women at any gestational age and new mothers of children under the age of 1 year were eligible for inclusion. The women were instructed to answer the questions related to their current or latest pregnancy. Detailed information regarding the recruitment tools used and internet penetration rates for individual countries have been described previously. $^{24}$
This substudy sample was restricted to women with residence in European countries only. Eligible countries were divided into three regions: (1) Western Europe: Austria, France, Italy, Switzerland, the Netherlands and the UK; (2) Northern Europe: Iceland, Finland, Norway and Sweden and (3) Eastern Europe: Croatia, Poland, Russia, Serbia and Slovenia. Unemployed women, women from European countries with $<100$ participants and women who did not answer the question 'Have you been on sick leave during this pregnancy?' were excluded (see online supplementary figure 1).

\section{Measures}

\section{Sick leave during pregnancy}

Our main outcome measure was sick leave during pregnancy. All women were asked to state whether or not they had been on sick leave during pregnancy (yes/no). Women answering 'yes' could also report the reason(s) for being on sick leave as free-text entries (see online supplementary table $\mathrm{S} 1$ ) and the trimester when the sick leave occurred (weeks 0-12, 13-24 and/or 25-delivery, which correspond to the first, second and third trimester, respectively). The rates of overall sick leave consist of women who reported to have been on sick leave in at least one trimester. Extension of sick leave referred to women on sick leave for more than one trimester.

Acute illnesses, chronic disorders and related medication use

Maternal illnesses and related medication use during pregnancy were the main independent variables in this study. Participants were presented with a list of nine common acute pregnancy-related illnesses (ie, common cold; constipation; heartburn and reflux problems; headache; NVP; other infections; pain in the neck, back or pelvic girdle; sleeping problems and urinary tract infection) and nine chronic disorders (ie, allergy, anxiety, asthma, depression, diabetes, epilepsy, hypothyroidism, cardiovascular and rheumatic disorders). In addition, the women could report as free text any other chronic disorders not previously listed. Women who reported that they had experienced any illnesses or disorders were asked to report any related medication use as a free-text entry. For the acute pregnancy-related illnesses, we focused on NVP, pain and sleeping problems because these illnesses were specifically stated as reasons for sick leave in pregnancy (figure 2). The four most prevalent chronic disorders were also studied, that is, mood disorders (depression and anxiety), asthma, allergy and hypothyroidism.

The questionnaire also included a list of common overthe-counter (OTC) medication groups (ie, analgesics, nasal spray/drops, antacids, antiemetics and laxatives) and participants could report whether these OTC medications were used. Women reporting any medication use were asked to specify the timing of usage according to pregnancy weeks (weeks 0-12, 13-24 and 25-delivery). Medication use did not include vitamins, mineral supplements and herbal or supplementary products.

Each country's sick leave policy category was also an independent variable of interest. The policies were 
categorised into 'high', 'medium', or 'low' based on the percentage of wage replacement during sick leave and the number of waiting days prior to wage replacement in each country. Information from 'Social Security Programs Throughout the World 2012' was used for the categorisation. ${ }^{25}$ The criteria were: 'high', full $(100.0 \%)$ wage replacement for everyone and no waiting days; 'medium', $60.0 \%-100.0 \%$ wage replacement and no waiting days; and 'low', $<100.0 \%$ wage replacement and 1-3 waiting days.

\section{Maternal sociodemographic and lifestyle factors}

Several maternal characteristics and health-related factors were assessed as potential confounders as they may be risk factors for sick leave in pregnancy and also be associated with medication use in pregnancy, the country of residence and, hence, the sick leave policy. These variables included age, maternal status (pregnant or mother at the time of answering the questionnaire), parity, marital status, employment status, highest level of education, folic acid use before and/or during pregnancy, smoking during pregnancy, alcohol consumption after awareness of pregnancy and whether or not the pregnancy was planned or secondary to infertility treatment. These variables were categorised as presented in table 1 .

\section{Ethics}

The South-East Regional Ethics Committee (REC) in Norway was notified about the main study and confirmed that the study did not require formal ethical approval because of anonymity. Additional ethical approval or study notification to the relevant national Ethics Boards was achieved in specific countries as required by the national legislation. Informed consent was considered given when the women answered 'Yes' to the question 'Are you willing to participate in the study?' before accessing the online questionnaire. All data were handled and stored anonymously.

\section{Statistical analysis}

Descriptive analyses on the prevalence of sick leave by the timing in gestation and country of residency, as well as reasons for being on sick leave were performed. The Chi-Squared test and the Fisher's exact tests were used to compare women's sociodemographic, lifestyle and pregnancy-related factors according to the overall prevalence of sick leave and the extension of sick leave during pregnancy. A univariate and multivariate generalised estimating equation with logit link function accounting for clustering on country level was used to estimate the association of: (1) maternal medication use and (2) sick leave policies with: (A) overall sick leave during pregnancy (yes vs. no) and (B) extension of sick leave in pregnancy (in one trimester only vs. in any two or all three trimesters). The associations were presented as crude odds ratios (OR) and adjusted OR (aOR) with the 95\% confidence interval (CI). Potential confounders were identified according to prior knowledge and current literature and by using directed acyclic graphs. The same set of confounders (ie, maternal age, parity, maternal status, marital status, education level, employment, infertility treatment, whether or not the pregnancy was planned, alcohol use in pregnancy, smoking in pregnancy, acute illnesses (other than the one of interest) and chronic disorders (other than the one of interest)), was used for all models containing medication use as the independent variable. Potential confounders for the 'sick leave policy' model were fitted by removing all variables having no role in the model, that is, variables yielding $<10 \%$ change in the beta coefficients of the 'sick leave policy' variable. As only new mothers had full overview of the pregnancy in relation to sick leave, a sensitivity analysis restricted to this group was conducted. Sensitivity analyses were also performed taking into account the differences in maternity leave policies in each country. These analyses were restricted to pregnant women who were not qualified for maternity leave (online supplementary table S2) when the electronic questionnaire was completed. A p value $<0.05$ was considered statistically significant. All statistical analyses were performed using the Data Analysis and Statistical Software Stata/MP v.14.

\section{RESULTS}

\section{Population characteristics}

A total of 9615 women replied to the informed consent question after reading the study description. Of these, $9483(98.7 \%)$ completed the online questionnaire. This subsample was restricted to $6686(69.5 \%)$ women from 12 European countries: Croatia, Finland, France, Italy, Norway, Poland, Russia, Serbia, Slovenia, Sweden, Switzerland and the UK. A flowchart of women who met the inclusion and exclusion criteria for this final study sample, along with the number of participants from each country is summarised in online supplementary figure 1. Maternal health and lifestyle factors, sick leave policy categorisations for each country and sociodemographic characteristics of the study sample in relation to sick leave are summarised in table 1 .

Fifty-two per cent $(n=3486)$ of the included women were pregnant at the time of accessing and answering the questionnaire, while the remaining were new mothers of children under the age of 1 year $(n=3200)$. Pregnant women were often younger and primiparous compared with new mothers (online supplementary table S3). The average gestation week (GW) among pregnant women was 23 (range 1-42), while almost half (48.5\%) of the new mothers had a child over 6 months of age. Detailed information regarding the maternal status and GW for each individual country is presented in online supplementary table S4.

\section{Sick leave during pregnancy}

A total number of 3385 (50.6\%) women had been on sick leave at some point in pregnancy, with a higher reported rate among new mothers $(55.3 \%)$ compared with pregnant women $(46.4 \%)$. The prevalence of sick leave in each individual country is presented in figure 1 . The 
Table 1 Maternal health and lifestyle factors, sociodemographic characteristics and sick leave policies in relation to sick leave in pregnancy

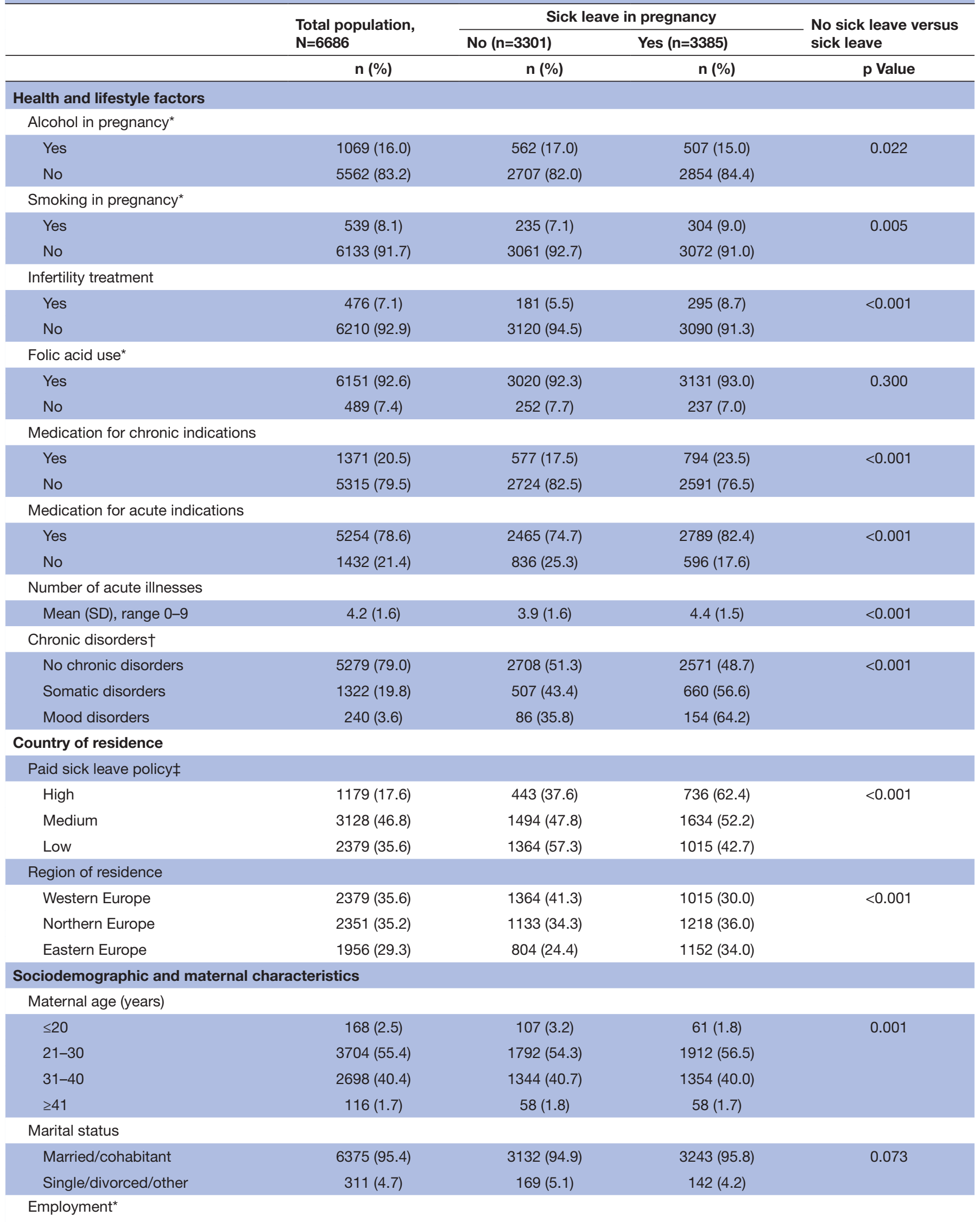


Table 1 Continued

\begin{tabular}{|c|c|c|c|c|}
\hline & \multirow{2}{*}{$\begin{array}{l}\text { Total population, } \\
\mathrm{N}=6686\end{array}$} & \multicolumn{2}{|c|}{ Sick leave in pregnancy } & \multirow{2}{*}{$\begin{array}{l}\text { No sick leave versus } \\
\text { sick leave }\end{array}$} \\
\hline & & No $(n=3301)$ & Yes $(n=3385)$ & \\
\hline & n (\%) & n (\%) & n (\%) & p Value \\
\hline Healthcare personnel & $1088(16.3)$ & $445(13.5)$ & $643(19.0)$ & \\
\hline Student & $695(10.4)$ & $492(14.9)$ & $203(6.0)$ & \\
\hline High school & $1736(26.0)$ & $845(25.6)$ & $891(26.3)$ & \\
\hline University or college & $3985(59.6)$ & $1994(60.4)$ & $1991(58.8)$ & \\
\hline Other education & $774(11.6)$ & $365(11.1)$ & $409(12.1)$ & \\
\hline \multicolumn{5}{|c|}{ Maternal status at the time of answering the questionnaire } \\
\hline Pregnant in third trimester & $1585(23.7)$ & $716(45.2)$ & $869(54.8)$ & \\
\hline \multicolumn{5}{|l|}{ Primiparous } \\
\hline Yes & 3603 (53.9) & $1830(55.4)$ & $1773(52.4)$ & 0.012 \\
\hline No & $3083(46.1)$ & $1471(44.6)$ & $1612(47.6)$ & \\
\hline \multicolumn{5}{|l|}{ Pregnancy planned* } \\
\hline Yes & 4657 (69.9) & $2229(67.7)$ & $2428(72.0)$ & 0.001 \\
\hline No, but expected & $1491(22.4)$ & 788 (23.9) & 703 (20.8) & \\
\hline No & $519(7.8)$ & 277 (8.4) & $242(7.2)$ & \\
\hline
\end{tabular}

${ }^{*}$ Total numbers do not add up due to missing values: folic acid, $n=46(0.7 \%)$; employment, $n=10(0.2 \%)$; alcohol in pregnancy, $n=55(0.8 \%)$; smoking in pregnancy, $\mathrm{n}=14(0.2 \%)$ and pregnancy planned, $\mathrm{n}=19(0.3 \%)$.

†Chronic disorders were categorised as no conditions, somatic conditions only (allergy, asthma, diabetes, epilepsy, hypothyroidism, cardiovascular diseases and rheumatic disorders) and any mood disorders (anxiety and/or depression). Women with both somatic and mood disorders were categorised in the latter group.

łHigh: Norway; medium: Croatia, Finland, Poland, Russia, Serbia, Slovenia and Sweden; low: Italy, France, the UK and Switzerland.

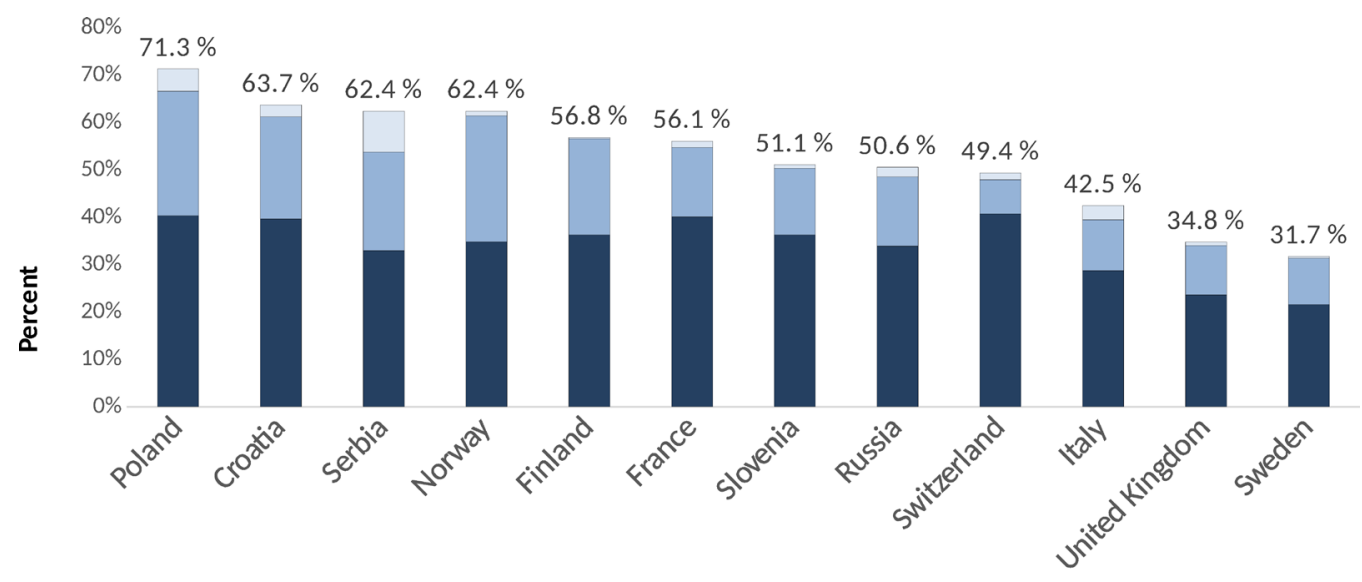

Country of residence

One trimester only $\square \geq 2$ trimesters $\square$ Unspecified Total \%

Figure 1 Sick leave rates in each participating country. The numbers of participants in each country were: Poland, $n=533$; Croatia, $n=237$; Serbia, $n=173$; Norway, $n=1179$; Finland, $n=438$; France, $n=287$; Slovenia, $n=135$; Russia, $n=878$; Switzerland, $\mathrm{n}=486$; Italy, $\mathrm{n}=720$; the UK, $\mathrm{n}=886$; and Sweden, $\mathrm{n}=734$. 


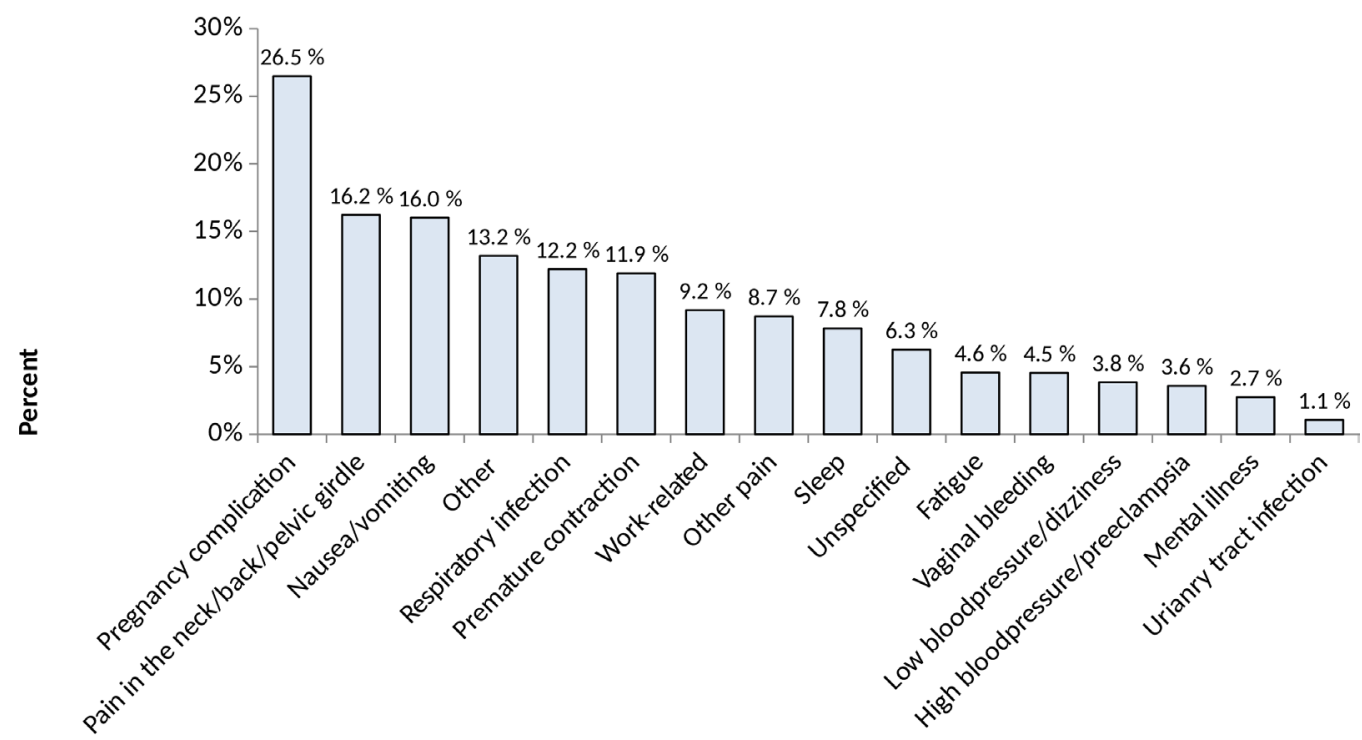

Reasons for sick leave in pregnancy

Figure 2 Reasons for sick leave in pregnancy $(n=3385)$. A woman could report several reasons for being on sick leave. More details on the sick leave categories are presented in online supplementary table S1.

proportions of women on sick leave in the first, second and/or third trimester were $38.5 \%, 48.1 \%$ and $52.1 \%$, respectively. Most women $(64.1 \%)$ had been on sick leave in one trimester only $(\mathrm{n}=2170)$, whereas $32.3 \%(\mathrm{n}=1095)$ had been on sick leave in any two or all three trimesters. Timing of sick leave for the remaining 120 women $(0.04 \%)$ was unknown.

The rates of overall prevalence and the extension of sick leave in each individual country are presented in online supplementary table S5. The most common reasons for being on sick leave were pregnancy complications $(26.0 \%)$; pain in the neck, back or pelvic girdle (16.2\%) and NVP (16.0\%) (figure 2). Reasons for sick leave were categorised into the 16 most prevalent categories as shown in figure 2. More details on the sick leave categories are presented in onlinesupplementary table S1.

Acute illnesses, chronic disorders and related medication use Women on sick leave had a significantly higher number of reported acute illnesses (mean (SD): 4.4 (1.5) vs 3.9 (1.6), $\mathrm{p}<0.001)$ and chronic disorders $(0.7(0.6)$ vs 0.2 $(0.5), \mathrm{p}<0.001)$ compared with women not on sick leave. The same trends were seen among women with extensions of sick leaves and medicated women compared with women who were sick leave in one trimester only and non-medicated women, respectively (data not shown).

Women medicated for NVP, pain or sleeping problems had significantly higher rates of sick leave due to the indication for medication use than non-medicated women

Table 2 Proportions of women on sick leave due to NVP, pain or sleeping problems according to medication use for the reported conditions

\begin{tabular}{lllll}
\hline & & \multicolumn{2}{c}{ Sick leave due to the reported condition } & Yes versus No \\
\cline { 3 - 5 } Conditions & Total & \multicolumn{1}{c}{ Yes } & No & p Value \\
\hline NVP & 4841 & $535(11.1)$ & $4306(89.0)$ & $<0.001$ \\
Medicated, $\mathrm{n}(\%)$ & $781(16.1)$ & $186(23.8)$ & $595(76.2)$ & \\
Non-medicated, $\mathrm{n}(\%)$ & $4060(84.9)$ & $349(8.6)$ & $3711(91.4)$ & \\
\hline Pain* & 5396 & $858(15.9)$ & $4538(84.1)$ & $<0.001$ \\
Medicated, $\mathrm{n}(\%)$ & $3320(62.0)$ & $631(19.0)$ & $2689(81.0)$ & \\
Non-medicated, $\mathrm{n}(\%)$ & $2076(38.0)$ & $227(10.9)$ & $1849(89.1)$ & \\
Sleeping problems & 3748 & $198(5.3)$ & $3550(94.7)$ & 0.016 \\
Medicated, $\mathrm{n}(\%)$ & $105(2.8)$ & $11(10.5)$ & $94(90.0)$ & \\
Non-medicated, $\mathrm{n}(\%)$ & $3643(97.2)$ & $187(5.1)$ & $3456(94.9)$ & \\
\hline
\end{tabular}

${ }^{*}$ Reasons for sick leave categorised as pain in the neck, back or pelvic girdle, other pain and headache. NVP, nausea and vomiting. 
Table 3 Associations with sick leave during pregnancy, presented as crude odds ratios (OR) and adjusted OR (aOR) with the 95\% confidence interval $(\mathrm{Cl})$.

Sick leave during pregnancy

In any two or all three trimesters versus any one trimester only

\section{Independent variables}

Yes versus no aOR $(95 \% \mathrm{Cl})$

\begin{tabular}{|c|c|c|c|c|c|}
\hline & Total, n & OR $(95 \% \mathrm{Cl})$ & aOR $(95 \% \mathrm{Cl})$ & OR $(95 \% \mathrm{Cl})$ & aOR $(95 \% \mathrm{Cl})$ \\
\hline \multicolumn{6}{|l|}{ Chronic disorders } \\
\hline Mood disorders ${ }^{\star}$ & 6446 & Reference & Reference & Reference & Reference \\
\hline No & 199 & 1.82 (1.36 to 2.43 ) & 2.05 (1.50 to 2.77 ) & 1.69 (1.17 to 2.43$)$ & 1.77 (1.21 to 2.58$)$ \\
\hline Asthma & 6369 & Reference & Reference & Reference & Reference \\
\hline Allergy & 6098 & Reference & Reference & Reference & Reference \\
\hline No & 339 & 1.55 (1.25 to 1.93$)$ & 1.51 (1.20 to 1.90$)$ & 1.28 (0.95 to 1.72$)$ & 1.38 (1.01 to 1.88$)$ \\
\hline $\begin{array}{l}\text { Yes, non-medicated } \\
\text { Yes, medicated }\end{array}$ & 249 & $1.62(1.26$ to 2.10$)$ & $1.49(1.14$ to 1.94$)$ & 1.51 (1.09 to 2.09$)$ & 1.50 (1.07 to 2.10$)$ \\
\hline \multicolumn{6}{|l|}{ Acute illnesses } \\
\hline Nausea and vomiting & 1836 & Reference & Reference & Reference & Reference \\
\hline No & 4060 & 1.27 (1.14 to 1.42$)$ & 2.49 (1.58 to 3.93$)$ & $1.26(1.05$ to 1.51$)$ & 1.95 (0.79 to 4.81$)$ \\
\hline $\begin{array}{l}\text { Yes, non-medicated } \\
\text { Yes, medicated }\end{array}$ & 790 & 2.29 (1.92 to 2.72$)$ & 4.52 (2.01 to 7.27$)$ & 2.30 (1.81 to 2.94$)$ & 3.69 (1.49 to 9.13$)$ \\
\hline Paint & 852 & Reference & Reference & Reference & Reference \\
\hline No & 2076 & 1.63 (1.39 to 1.91$)$ & 1.07 (0.85 to 1.36$)$ & 1.42 (1.05 to 1.92$)$ & 1.03 (0.66 to 1.60$)$ \\
\hline $\begin{array}{l}\text { pain, non-medicated } \\
\text { Pain, medicated }\end{array}$ & 3.758 & 2.09 (1.80 to 2.43$)$ & $1.38(1.11$ to 1.71$)$ & 1.96 (1.46 to 2.62$)$ & $1.38(0.91$ to 2.10$)$ \\
\hline Sleeping problems (SP) & 2938 & Reference & Reference & Reference & Reference \\
\hline
\end{tabular}

Missing data $<5 \%$, as presented in table 1 .

${ }^{*}$ Depression and/or anxiety.

†Pain in the neck, back or pelvic girdle or headache.

with the same conditions (table 2). These women had also higher rates of sick leave due to any reason (data not shown).

\section{Sick leave policies}

The categorisation of countries according to sick leave policies was as follows: 'high'-Norway $(n=1179)$; 'medium'-Croatia, Finland, Poland, Russia, Serbia, Slovenia and Sweden ( $\mathrm{n}=3128)$; and 'low'-Italy, France, the UK and Switzerland $(\mathrm{n}=2379)$. Women from Norway with 'high' sick leave policies had the highest overall prevalence $(62.4 \%)$ and extensions of sick leave $(43.2 \%)$.

\section{Factors associated with sick leave}

Having chronic asthma, allergy, hypothyroidism or mood disorders was positively associated with sick leave in pregnancy regardless of medication use (table 3). Women who did not report any treatment (non-medicated) for asthma, allergy or hypothyroidism had a higher likelihood (1.5-fold to 2.7-fold) of being on sick leave compared with those without the disorder. These associations were greater than for medicated women (1.3-fold to 1.5-fold), whereas the inverse was observed in relation to mood disorders (non-medicated vs medicated aOR 2.1 vs 3.1). The association between medicated acute illnesses and sick leave was also greater than for non-medicated acute illnesses and sick leave, when compared with no acute illness (table 3). Women from countries with 'low' sick leave policies were less likely to have extensions of sick leaves in pregnancy compared with women from countries with 'medium' sick leave policies (aOR $0.63,95 \%$ CI 0.49 to 0.82 ).

\section{Sensitivity analysis}

The sensitivity analyses addressing the differences in maternity leave policies in each country showed that the 
magnitude of the association between having a medicated condition and sick leave did not substantially differ from the main analyses (data not shown).

In the sensitivity analysis restricted to new mothers, the magnitudes of the associations between having a medicated condition and sick leave were generally similar to those of the main analysis $( \pm 20 \%$ change of the point estimates), with the exception of medicated mood disorders ( $+57 \%$ change) and non-medicated NVP (>35\% lower) (data not shown).

These sensitivity analyses could not be done for hypothyroidism and sleeping problems due to small sample sizes.

\section{DISCUSSION}

To our knowledge, this is the first study investigating patterns of sick leave in pregnancy on a multinational level, focusing on maternal medication use. The results indicate that the rates of sick leave in pregnancy vary greatly within Europe, ranging from $31.7 \%$ in Sweden to $71.3 \%$ in Poland. The most common reason for sick leave was pregnancy complications, but this differed according to the country and region of residence. When compared with women without acute illnesses, women using medications for acute illnesses were 1.4-fold to 5.4-fold more likely to be on sick leave; while non-medicated women were 1.1-fold to 3.1-fold more likely. The opposite trend was seen for the chronic disorders such as asthma, allergy and hypothyroidism. Residence in countries with 'low' sick leave policies seems to decrease the likelihood of extending sick leaves.

Eastern European women had the highest rates of work-related sick leave; on the other hand, they also had the lowest rates of sick leave due to pain and NVP (online supplementary table S5). These findings may reflect differences in women's perception towards their own health $^{20}$ and the need for sick leave during pregnancy. Women on long-term sick leave seem to have a lower self-rated health and lower quality of life compared with women not on sick leave or on short-term sick leave. ${ }^{20}$ Studies have also shown the practice of prescribing sick leave varies greatly. ${ }^{14}$ Clinical specialists tend to prescribe shorter sick leaves than general practitioners and more sick days are often prescribed in smaller municipalities than larger ones. ${ }^{13}$ We had no information regarding the prescribing physician, the number of sick days prescribed, nor if the women were from a suburban or urban area; thus, prescribing practices may have contributed to the different patterns of sick leave seen in this study.

The magnitude of the association between non-medicated chronic disorders (ie, asthma, allergy and hypothyroidism) and sick leave was greater than the association for medicated chronic disorders, when both groups were compared with women having no chronic disorder. Yet, the opposite trend was seen for all the acute illnesses investigated as well as for chronic mood disorders. Previous studies have shown that acute pregnancy-related illnesses can have a huge impact on pregnant women's daily activities, such as walking, standing or changing position, as well as quality of life. ${ }^{32627}$ On the other hand, perinatal mood disorders can have significant detrimental effects on the mother, child and whole family. ${ }^{28}$ A possible explanation for our findings is that women medicated for acute illnesses or for treatment of mood disorders had a more severe condition; hence, they were more disabled than non-medicated women. Indeed, pharmacological treatment with antidepressants in pregnancy is usually reserved for women with a major mood disorder, or as a second-line therapy when non-pharmacological therapies have failed. ${ }^{29}$ Thus, medication use for acute illnesses as well as for mood disorders may be a proxy for the severity of the conditions. However, studies have shown that common pregnancy-related illnesses, such as NVP, are often mismanaged and neglected by healthcare personnel. ${ }^{26}$ The results of our study may support these findings as the magnitude of the association between non-medicated and medicated acute illnesses, specifically for NVP and sleeping problems, was greater than that for chronic disorders. Reducing sick leave rates among pregnant women is beneficial for public health and has major economic interest for society. Previous research has mainly focused on pre-existing chronic disorders in pregnancy, and these women are generally followed-up adequately. However, our study indicates that there is a need to focus on other aspects in pregnancy. Therefore, future research should investigate whether or not sick leave among pregnant women can be prevented by optimising management of acute pregnancy-related illnesses.

Our results suggest that sick leave policies may have an impact on both the rates and the extensions of sick leave, especially in countries with limited benefits. A 'high' sick leave policy was not significantly associated with increased rates of sick leave compared with 'medium' policies. Interestingly, 'low' sick leave policies seem to decrease the likelihood of extending of sick leaves in pregnancy even after adjusting for maternal characteristics and sociodemographic and lifestyle factors. These findings are consistent with a report from WHO, which found countries with a medium scope of benefits had the highest number of sick leave days in Europe. ${ }^{15}$ However, there was no doubt that countries with limited benefits had the lowest rates of sick leave, like in our study.

We found that the majority of women were on sick leave during the last trimester, and this is consistent with a study conducted in Sweden. ${ }^{2}$ However, in countries like Russia and the UK, maternity leave can be taken in the beginning of the third trimester, which may explain the relatively low rates of sick leave seen in these countries compared with the other countries in this study. However, as shown in the sensitivity analyses restricted to pregnant women who were not qualified to receive maternity leave in each country, the associations between maternal conditions and sick leave did not considerably differ.

The main strength of this study was the large sample size and the uniform data collection methodology used 
across all participating countries, allowing intercountry comparisons of sick leave in pregnancy. The precision of our estimates of sick leave was within $\pm 5.0 \%$ in each country with the exception of France, Croatia, Serbia and Slovenia where the precision ranged from $\pm 6 \%$ to $8 \%$. We had detailed information regarding maternal health, including medication use and comorbidities, which could be risk factors for sick leave in pregnancy. In addition, we considered the differences in sick leave policies across the participating countries using external sources. Our previous studies also demonstrated that the study samples were sufficiently representative of the general birthing population in each individual country with regards to age and smoking habits. However, they had on average higher levels of education and were more likely to be primiparous. ${ }^{24}$

The limitations of this study include the lack of detailed information regarding sick leave in pregnancy, that is, exact timing, duration and if it was part time or full time. Also, we were not able to distinguish between sick leave among women employed in private and public sectors, and this could have yielded another sick leave pattern. Moreover, data were collected via an online questionnaire ; hence, a conventional response rate could not be calculated. However, there are examples of web-based recruitment methods that show reasonable validity in epidemiology studies. ${ }^{30} 31$ Furthermore, the web-recruitment approach could introduce the possibility of selection bias as only women with internet access who visited the web pages where the invitation to participate was posted could participate. However, some studies have shown that a large proportion of pregnant women tend to use the internet, including discussion forums and social networks, when in need of pregnancy information. ${ }^{3233}$ Also, internet access in households in our European target population were generally high in 2012, ranging from $63.0 \%$ in Italy to $93.0 \%$ in Norway. ${ }^{34}$ Another limitation of our study is that all data were self-reported and therefore depended on the women's perception and recall rather than validated data. The use of medications and sick leave during pregnancy may have been underestimated due to poorer recall, especially among women who were not pregnant at the time of answering the questionnaire. A previous study demonstrated that retrospective, self-reported sick leave data due to musculoskeletal diseases were comparable with registered sick leave. ${ }^{35}$ However, other studies have reported discrepancies in sick leave rates in self-reported data when compared with register data, especially for long-term sick leave. ${ }^{36} 37$ The inclusion of pregnant women at any gestation may also have underestimated the rates of sick leave, as women in early gestation may not have been on sick leave at the time of answering the questionnaire, but were later on in pregnancy. The sensitivity analyses restricted to new mothers only showed that the magnitude of the association between medication use for NVP and sick leave was reduced and no longer significant. This may be because women reported current illnesses to a larger degree and NVP often occur in the beginning of the pregnancy. Also, the French and the Russian study samples represented a small proportion of the general birthing population in these countries; hence, the generalisability of our results should be interpreted with caution, especially in those countries.

\section{CONCLUSION}

A large proportion of women were on sick leave during pregnancy, but the rates varied greatly across European countries. Maternal medication use was associated with sick leave, especially for acute illnesses. The differences in sick leave patterns across European countries only partially reflect the differences in each country's sick leave policy, which implies that sick leave in pregnancy is also affected by other national differences.

Acknowledgements The authors thank the Organisation of Teratology Information Services, the European Network of Teratology Information Services Scientific Board and website providers who contributed to the recruitment phase of the main study "Medication Use in Pregnancy: a cross-sectional, multinational web-based study". They also thank all study national coordinators (Twigg MJ, Zagorodnikova K, Mårdby AC, Moretti ME, Drozd M, Panchaud A, Hameen-Anttila K, Rieutord A, Gjergja Juraski R, Odalovic M, Kennedy D, Rudolf G, Juch H, Passier JLM and Bjornsdottir I). They are grateful to all the women who took part in this study.

Contributors $\mathrm{AL}$ and $\mathrm{HN}$ : conceived the idea and participated in the design and coordination of the main study. BTT: performed the initial analysis and drafted the first manuscript. AL: contributed substantially to the final analysis for this substudy. AL, PK and HN: contributed to the interpretation of the results and critically revised the manuscript for important intellectual content. All authors: read and approved the final version of the manuscript and are in agreement to be accountable for all aspects of the work.

Funding The main study "Medication Use in Pregnancy: a cross-sectional, multinational web-based study" was supported by the Foundation for Promotion of Norwegian Pharmacies and the Norwegian Pharmaceutical Society. AL postdoctoral research fellowship is funded through the HN's ERC Starting Grant "DrugsInPregnancy" grant number 678033.

\section{Competing interests None declared.}

Ethics approval The South-East Regional Ethics Committee (REC) in Norway was notified about the main study and confirmed that the study did not require formal ethical approval. Additional ethical approval or study notification to the relevant national Ethics Boards was achieved in specific countries as required by the national legislation.

Provenance and peer review Not commissioned; externally peer reviewed.

Data sharing statement № additional data are available. Researchers can apply for data access for subprojects within the overall aims of the main study: "The Multinational Medication Use in Pregnancy Study".

Open Access This is an Open Access article distributed in accordance with the Creative Commons Attribution Non Commercial (CC BY-NC 4.0) license, which permits others to distribute, remix, adapt, build upon this work non-commercially, and license their derivative works on different terms, provided the original work is properly cited and the use is non-commercial. See: http://creativecommons.org/ licenses/by-nc/4.0/

(C) Article author(s) (or their employer(s) unless otherwise stated in the text of the article) 2017. All rights reserved. No commercial use is permitted unless otherwise expressly granted.

\section{REFERENCES}

1. Sydsjö A, Sydsjö G, Kjessler B. Sick leave and social benefits during pregnancy: a Swedish-Norwegian comparison. Acta Obstet Gynecol Scand 1997;76:748-54. 
2. Mogren I, health P. Perceived health, sick leave, psychosocial situation, and sexual life in women with low-back pain and pelvic pain during pregnancy. Acta Obstet Gynecol Scand 2006;85:647-56.

3. Malmqvist S, Kjaermann I, Andersen K, et al. The association between pelvic girdle pain and sick leave during pregnancy; a retrospective study of a Norwegian population. BMC Pregnancy Childbirth 2015;15:237.

4. Dørheim SK, Bjorvatn B, Eberhard-Gran M. Sick leave during pregnancy: a longitudinal study of rates and risk factors in a Norwegian population. BJOG 2013;120:521-30.

5. Robinson HS, Eskild A, Heiberg E, et al. Pelvic girdle pain in pregnancy: the impact on function. Acta Obstet Gynecol Scand 2006;85:160-4.

6. Heitmann K, Holst L, Lupattelli A, et al. Treatment of nausea in pregnancy: a cross-sectional multinational web-based study of pregnant women and new mothers. BMC Pregnancy Childbirth 2015;15:321.

7. Sivertsen B, Hysing M, Dørheim SK, et al. Trajectories of maternal sleep problems before and after childbirth: a longitudinal populationbased study. BMC Pregnancy Childbirth 2015;15:129.

8. Khojasteh F, Arbabisarjou A, Boryri T, et al. The relationship between maternal employment status and pregnancy outcomes. Glob J Health Sci 2016;8:53533.

9. Xu G, Wu Y, Yang L, et al. Risk factors for early miscarriage among Chinese: a hospital-based case-control study. Fertil Steril 2014;101:1663-70.

10. Axelsson G, Rylander R, Molin I. Outcome of pregnancy in relation to irregular and inconvenient work schedules. $\mathrm{Br} \mathrm{J}$ Ind Med 1989;46:393-8.

11. Bodin L, Axelsson G, Ahlborg G. The association of shift work and nitrous oxide exposure in pregnancy with birth weight and gestational age. Epidemiology 1999;10:429-36.

12. Hansen ML, Thulstrup AM, Juhl M, et al. Occupational exposures and sick leave during pregnancy: results from a Danish cohort study. Scand J Work Environ Health 2015;41:397-406.

13. Kankaanpää AT, Franck JK, Tuominen RJ. Variations in primary care physicians' sick leave prescribing practices. Eur J Public Health 2012;22:92-6.

14. Rudbeck M. Variation in patients' sick leave between general practitioner practices. Scand J Public Health 2014;42:621-6.

15. Scheil-Adlung $X$, Sandner $L$. The case for paid sick leave - Word Health Report Background Paper No. 9. 2010 http://www.who.int/ healthsystems/topics/financing/healthreport/SickleaveNo9FINAL. pdf?ua=1 (accessed: October 2016).

16. Nordeng $\mathrm{H}$, Elseviers $\mathrm{M}$, Wettermakr B, et al. Drug utilization in pregnant women. Drug Utilization Research Methods and Applications. West Sussex: Wiley-Blackwell, 2016:240-7.

17. Nordeng $H$, Ystrøm E, Einarson A. Perception of risk regarding the use of medications and other exposures during pregnancy. Eur J Clin Pharmacol 2010;66:207-14.

18. Petersen I, McCrea RL, Lupattelli A, et al. Women's perception of risks of adverse fetal pregnancy outcomes: a large-scale multinational survey. BMJ Open 2015;5:e007390.

19. Dekkers-Sánchez PM, Hoving JL, Sluiter JK, et al. Factors associated with long-term sick leave in sick-listed employees: a systematic review. Occup Environ Med 2008;65:153-7.
20. Eriksson HG, von Celsing AS, Wahlström R, et al. Sickness absence and self-reported health a population-based study of 43,600 individuals in Central Sweden. BMC Public Health 2008;8:426.

21. Kristensen P, Nordhagen R, Wergeland $E$, et al. Job adjustment and absence from work in mid-pregnancy in the Norwegian mother and Child Cohort Study (MoBa). Occup Environ Med 2008;65:560-6.

22. Strand K, Wergeland E, Bjerkedal T, load W. Work load, job control and risk of leaving work by sickness certification before delivery, Norway 1989. Scand J Soc Med 1997;25:193-201.

23. Werner EL, Cote P. Low back pain and determinants of sickness absence. Eur J Gen Pract 2009;15:74-9.

24. Lupattelli $A$, Spigset $O$, Twigg MJ, et al. Medication use in pregnancy: a cross-sectional, multinational web-based study. BMJ Open 2014;4:e004365

25. Social Security Administration and the International Social Security Association. Social Security Programs throughout the world: Europe 2012. https://www.ssa.gov/policy/docs/progdesc/ssptw/2010-2011/ europe/ssptw10europe.pdf (accessed June 2016).

26. Heitmann K, Svendsen HC, Sporsheim IH, et al. Nausea in pregnancy: attitudes among pregnant women and general practitioners on treatment and pregnancy care. Scand J Prim Health Care 2016;34:13-20

27. Rezaei E, Moghadam ZB, Saraylu K. Quality of life in pregnant women with sleep disorder. J Family Reprod Health 2013;7:87-93.

28. Howard LM, Molyneaux E, Dennis CL, et al. Non-psychotic mental disorders in the perinatal period. Lancet 2014;384:1775-88.

29. Hendrick V, Psychiatric disorders in Pregnancy and the Postpartum: principles and treatment. Editor. Totawa, New Jersey: Humana Press, 2006.

30. Ekman A, Dickman PW, Klint A, et al. Feasibility of using web-based questionnaires in large population-based epidemiological studies. Eur J Epidemiol 2006;21:103-11.

31. Huybrechts KF, Mikkelsen EM, Christensen T, et al. A successful implementation of e-epidemiology: the Danish pregnancy planning study 'Snart-Gravid'. Eur J Epidemiol 2010;25:297-304.

32. O'Higgins A, Murphy OC, Egan A, et al. The use of digital media by women using the maternity services in a developed country. Ir Med $J$ 2014;107:313-5.

33. Bert F, Gualano MR, Brusaferro S, et al. Pregnancy e-health: a multicenter Italian cross-sectional study on Internet use and decision-making among pregnant women. J Epidemiol Community Health 2013;67:1013-8.

34. Internet use in households and by individuals in 2012. Eurostat Statistics in focus 2012 http://ec.europa.eu/eurostat/documents/ 3433488/5585460/KS-SF-12-050-EN.PDF (accessed on October 2016).

35. Fredriksson $\mathrm{K}$, Toomingas $\mathrm{A}$, Torgén $\mathrm{M}$, et al. Validity and reliability of self-reported retrospectively collected data on sick leave related to musculoskeletal diseases. Scand J Work Environ Health 1998;24:425-31.

36. Stapelfeldt CM, Jensen C, Andersen NT, et al. Validation of sick leave measures: self-reported sick leave and sickness benefit data from a Danish national register compared to multiple workplace-registered sick leave spells in a Danish municipality. BMC Public Health 2012;12:661.

37. Pole JD, Franche RL, Hogg-Johnson S, et al. Duration of work disability: a comparison of self-report and administrative data. Am J Ind Med 2006;49:394-401. 